\title{
Revue sur les analogies et les différences relevées entre un cône de houblon et une baie de raisin : arômes soufrés et azotés
}

\author{
Marie-Lucie Kankolongo Cibaka, Jacques Gros, Sonia Collin
}

Université catholique de Louvain. Faculté des Bioingénieurs. Unité de Brasserie et des Industries Alimentaires. Earth and Life Institute. ELIM. Croix du Sud, 2 L7.05.07. BE-1348 Louvain-la-Neuve (Belgique).E-mail : sonia.collin@uclouvain.be

Reçu le 8 avril 2016, accepté le 19 septembre 2016.

Cet article est distribué suivant les termes et les conditions de la licence CC-BY (http://creativecommons.org/licenses/by/4.0/ deed.fr)

Introduction. Plusieurs similitudes peuvent être relevées entre le cône de houblon et la baie de raisin. Nous nous focaliserons dans cet article sur leurs arômes soufrés et azotés.

Littérature. Bien qu'initialement développées sur la vigne, les recherches sur les thiols polyfonctionnels ont abouti à l'identification d'un grand nombre d'analogues odorants dans le houblon. Parmi ceux-ci, le 3-sulfanylhexan-1-ol (3SHol) caractérisé par des arômes de pamplemousse ou de fruit de la passion est retrouvé à la fois dans le cône de houblon (72-117 $\mu \mathrm{g}$. $\mathrm{kg}^{-1}$ ) et dans le moût de raisin (66-121 ng. $\left.\mathrm{l}^{-1}\right)$. Des concentrations souvent au-dessus du seuil de perception sont atteintes dans la bière (jusque $200 \mathrm{ng} \cdot \mathrm{l}^{-1}$ ) ou dans le vin (jusque $6000 \mathrm{ng} \cdot \mathrm{l}^{-1}$ ), suite à la dégradation de précurseurs cystéinés et glutathionés durant la fermentation. Le cône de houblon renferme également de nombreux thiophènes et épisulfures d'origine terpénique, ainsi que des sulfures, polysulfures et thioesters aux arômes d'oignon, de verdure ou de fromage. Par contre, la présence de méthoxypyrazines n'est relevée à ce jour que dans le raisin.

Conclusions. Plusieurs analogies sont relevées entre les thiols polyfonctionnels du cône de houblon et de la baie de raisin. Les thiophènes, épisulfures, sulfures, polysulfures et thioesters sont principalement retrouvés dans le cône de houblon, alors que les méthoxypyrazines ne sont observées que dans le raisin.

Mots-clés. Raisin, vigne, cône, houblon, bière, vin, thiols, pyrazine, flaveur, fermentation alcoolique.

Similarities and differences between hop cones and grape berries: sulfur and nitrogen aromas. A review

Introduction. Similarities can be found between the flavors of hop cones and grapes. The present paper will focus on their sulfur and nitrogen odorants.

Literature. Although initially investigated in vines, recent research has led to the identification of a larger number of polyfunctional thiols in hop cones. These have included the pleasant-smelling grapefruit- or passion fruit-like 3-sulfanylhexan1-ol. Surprisingly, to date, the occurrence of sulfides, polysulfides and thioesters (onion, vegetable or cheese-like flavors), as well as various thiophenes and terpenic episulfides has been described extensively only in the hop literature, while methoxypyrazines have been described only in vine cultivars.

Conclusions. Among the sulfur and nitrogen odorants, many similarities have been observed between hop and grape polyfunctional thiols. Thiophenes, episulfides, sulfides, polysulfides and thioesters are mainly found in hop cones, while methoxypyrazines have been described only in grape.

Keywords. Grapes, vine, cones, hops, beers, wines, thiols, pyrazines, flavour, alcoholic fermentation.

\section{INTRODUCTION}

Cette synthèse bibliographique poursuit la description comparative du cône de houblon et de la baie de raisin. Une première étude axée sur le mode de culture du houblon et de la vigne et leur anatomie, ainsi que sur les tendances actuelles en matière de sélection est parue en 2013 (Gros et al., 2013a). Les constituants chimiques majeurs de ces deux matrices ont été décrits par Gros et al. (2013b) et de nombreux dérivés terpéniques et autres arômes oxygénés communs au houblon et à la vigne ont fait l'objet d'études menées par Kankolongo et al. (2014). Dans le présent article, nous nous focaliserons sur leurs molécules soufrées et azotées. Les thiols polyfonctionnels constituent probablement le plus bel exemple de complémentarité de recherche 
que l'on ait exploité ces dernières années entre le vin et la bière. Bien qu'initialement développées sur la vigne, ces recherches ont abouti à l'identification d'un grand nombre d'analogues odorants dans le houblon. Le houblon renferme également de nombreux sulfures et polysulfures, ainsi que des thioesters aux arômes de fromage. Nous discuterons ensuite de la présence de méthoxypyrazines dans certains cépages vinicoles, molécules encore jamais identifiées à ce jour dans un houblon.

\section{LES THIOLS POLYFONCTIONNELS}

Les thiols polyfonctionnels (mercaptans associés à une autre fonction chimique) ont été particulièrement bien étudiés dans la matrice "vin» depuis une trentaine d'années. Ils contribuent au bouquet de nombreux vins blancs et rosés, générant des arômes très caractéristiques pour certains d'entre eux (e.g. vins de Sauvignon Blanc) malgré leur présence toujours à l'état de traces (Tableau 1). Nous savons aujourd'hui qu'ils sont principalement issus de la dégradation de précurseurs cystéinés et glutathionnés présents dans le raisin. Nous ne reparlerons pas ici des mécanismes mis en jeu dans leur synthèse et leur hydrolyse puisqu'ils ont fait l'objet d'une revue bibliographique récente (Gros et al., 2013c).

\subsection{Les sulfanylalkyl alcools}

Dans la baie de raisin, aucun thiol polyfonctionnel libre n'a été quantifié. Cependant, le 3-sulfanylhexan1-ol (3SHol ; arômes de pamplemousse ou de fruit de la passion, respectivement pour les énantiomères $\mathrm{R}$ et $\mathrm{S}$; figure $\mathbf{1}(\mathbf{2 3})$ ) est retrouvé dans le mout de raisin à des teneurs de 66-121 ng. $\mathrm{l}^{-1}$, dans le cas du Sauvignon Blanc (Capone et al., 2010). La teneur en précurseur cystéiné du 3SHol peut atteindre 1, 22-111, 24 et $60 \mu \mathrm{g} \cdot \mathrm{l}^{-1}$ respectivement dans les mouts de Melon Blanc, Sauvignon Blanc, Riesling et Gewurztraminer, alors que le précurseur glutathioné y est retrouvé jusqu'à 0,$2 ; 4,4 ; 1,3$ et 5,9 $\mu \mathrm{g} \cdot \mathrm{l}^{-1}$ (Roland et al., 2010 ; Allen et al., 2011). Soixante pourcents des précurseurs cystéinés sont localisés dans la peau alors que les précurseurs glutathionés sont équitablement répartis entre la peau et la pulpe (Murat et al., 2001 ; Roland et al., 2011). Le seuil de perception du 3 SHol dans une solution hydroalcoolique $(12 \% \mathrm{v} / \mathrm{v})$ est de $50 \mathrm{ng} \cdot \mathrm{l}^{-1}$ pour la forme R et de $60 \mathrm{ng} \cdot 1^{-1}$ pour la forme S (Tominaga et al., 2006). Sa concentration peut atteindre $6000 \mathrm{ng}$. $1^{-1}$ dans les vins de Sémillon (Sauternes, tableau 1).

Le 4-sulfanyl-4-méthylpentan-2-ol (4S4M2Pol; arôme de pamplemousse, de raisin ; Figure 1(18)) a été retrouvé dans les vins de Sauvignon Blanc à des teneurs de 15-150 ng. $\mathrm{l}^{-1}$. Son seuil de perception est de $55 \mathrm{ng} \cdot \mathrm{l}^{-1}$ dans une solution hydroalcoolique $(12 \% \mathrm{v} / \mathrm{v})$ (Tominaga et al., 1998a). Le précurseur cystéiné de cet alcool a été identifié dans le mout de Sauvignon Blanc (Tominaga et al., 1998b).

Enfin, le 3-sulfanyl-3-méthylbutan-1-ol (3S3MBol ; arôme de légume cuit, d'oignon ; Figure 1(17)) a également été retrouvé dans les vins de Sauvignon Blanc, à des teneurs de 20-150 ng. $\mathrm{l}^{-1}$. Son seuil de perception étant plus élevé (1500 ng.l${ }^{1}$ ), ce composé participera peu au profil aromatique de ces vins, comparativement aux deux précédents (Tominaga et al., 1998a). Aucun article ne mentionne l'existence de son précurseur cysteiné (la félinine), alors qu'il a été caractérisé par Starkenmann et al. (2007 ; 2015) dans le Poncirus trifoliata L. et l'urine de chat.

Tableau 1. Teneurs en thiols volatils $\left(\mathrm{ng} \cdot \mathrm{l}^{-1}\right)$ quantifiés dans les vins de différents cépages (d'après Tominaga et al., $2000)$ - Content of volatile thiols $\left(n g \cdot l^{-1}\right)$ in wines of different grape varieties (according to Tominaga et al., 2000).

\begin{tabular}{llllll}
\hline Composé & 4S4M2Pone & 4S4M2Pol & 3S3MBol & 3SHA & 3SHol \\
\hline Gewürztraminer & $0,7-15$ & $0-14$ & $137-1322$ & $0-6$ & $40-3300$ \\
\hline Riesling & $0-9$ & $0-3$ & $26-190$ & $0-15$ & $123-1234$ \\
Muscat d'Alsace & $9-73$ & $0-45$ & $19-326$ & $0-1$ & $100-1800$ \\
\hline Pinot Gris & $0-3$ & $0-0,5$ & $21-170$ & $0-51$ & $312-1042$ \\
\hline Pinot Blanc & $0-1$ & 0 & $2-83$ & 0 & $88-248$ \\
\hline Sylvaner & $0-0,5$ & 0 & $1-99$ & 0 & $59-554$ \\
\hline Colombard & 0 & 0 & 0 & $20-60$ & $400-1000$ \\
\hline Petit Manseng (Jurançon) & 0 & 0 & $40-140$ & $0-100$ & $800-4500$ \\
\hline Sémillon (Sauternes) & 0 & 0 & $100-500$ & 0 & $1000-6000$ \\
\hline
\end{tabular}

4S4M2Pone : 4-sulfanyl-4-méthylpentan-2-one - 4-sulfanyl-4-methylpentan-2-one ; 4S4M2Pol : 4-sulfanyl-4-méthylpentan2-ol - 4-sulfanyl-4-methylpentan-2-ol ; 3S3MBol : 3-sulfanyl-3-méthylbutan-1-ol - 3-sulfanyl-3-methyl-butan-1-ol ; 3SHA :

3-sulfanylhexyl acétate - 3-sulfanylhexyl acetate ; 3SHol : 3-sulfanylhexan-1-ol - 3SHol, 3-sulfanylhexan-1-ol. 
<smiles>[R]CCCS</smiles>
$\mathrm{R}=\mathrm{OH}$ (14)<smiles>[R]CC(C)CS</smiles><smiles>[R]C(CC)CCS</smiles>

$\mathrm{R}=$ Acétate (7)

$\mathrm{R}=\mathrm{OH}$ (19)

$\mathrm{R}=\mathrm{O}=(31)$<smiles>[R]CC(CC)CS</smiles>

$\mathrm{R}=$ Acétate (10) $\mathrm{R}=\mathrm{O}=(30)$<smiles>[R]CCC(S)CCC</smiles>

$\mathrm{R}=$ Acétate (11) $\mathrm{R}=\mathrm{OH}$ (23) $\mathrm{R}=\mathrm{O}=(32)$<smiles>[R]CC(C)C(S)CC</smiles>
$=\mathrm{OH}(27)$ $\mathrm{R}=\mathrm{O}=(35)$<smiles>[R]CCC(S)CCCC</smiles><smiles>[R]CCS</smiles><smiles>CC(C)=CCS</smiles><smiles>[R]CCC(C)S</smiles><smiles></smiles><smiles>[R]CCC(S)CC</smiles><smiles>[R]CCC(S)C(C)C([R])[R]([R])=O</smiles><smiles>[R]CC(CS)CCCC</smiles><smiles>OCCCCCS</smiles><smiles>OCCCC1(S)CC1</smiles>

Figure 1. Structures de quelques thiols polyfonctionnels retrouvés dans le raisin, le cône de houblon, le vin ou la bière - Structures of some polyfunctional thiols found in grapes, hop cones, wine or beer.

(1) 3SPrA, (2) 1S3BA, (3) 3SBA, (4) 3S2MPrA, (5) 3S3MBA, (6) 4S4M2PA, (7) 1S3PA, (8) 3S2MBA, (9) 3SPA, (10) 3S2EPrA, (11) 3SHA, (12) 3S4MPA, (13) 3SOA, (14) 3SProl, (15) 3SBol, (16) 3S2MProl, (17) 3S3MBol, (18) 4S4M2Pol, (19) 1S3Pol, (20) 3S2MBol, (21) 3SPol, (22) 3S2MPol, (23) 3SHol, (24) 3S2BProl, (25) 3SHptol, (26) 3S4MPol, (27) 3SOol, (28) 3SPal, (29) 4S4M2Pone, (30) 3S2EPral, (31) 1S3Pone, (32) 3SHal, (33) 3SHptal, (34) 3S2BPral, (35) 3SOal, (36) 2SEol, (37) MBT, (38) 2SEA, (39) 3S2MBT, (40) 6SHol, (41) 4S4MPol ; Les noms correspondant aux abréviations sont présentés aux tableaux 2 - The corresponding chemical names are given in tables 2.

Un beaucoup plus grand nombre de sulfanylalkyl alcools ont été identifiés et quantifiés dans le cône de houblon, à des teneurs totales allant de 2 à $160 \mu \mathrm{g} \cdot \mathrm{kg}^{-1}$ (Tableau 2a ; Gros et al., 2011). Le 3-sulfanylhexan1-ol (3SHol) est le seul sulfanylalkyl alcool identifié actuellement à la fois dans le raisin et dans le cône de houblon (Kishimoto et al., 2008b ; Capone et al., 2010 ; Gros et al., 2011). Sous ses formes libre et liée, le 3SHol est une caractéristique de la variété Cascade où il est présent à des teneurs élevées (72$117 \mu \mathrm{g} \cdot \mathrm{kg}^{-1} \mathrm{du}$ thiol libre) comparativement à celles des cultivars Tomahawk, Nelson Sauvin, Nugget et Saaz (2-17 $\mu \mathrm{g} \cdot \mathrm{kg}^{-1}$; Gros et al., 2011). Les teneurs en 3 SHol du Cascade sont toutefois similaires à celles retrouvées sous forme libre dans les variétés Simcoe, Topaz et Fuggle (Kishimoto et al., 2008b). Dans la 


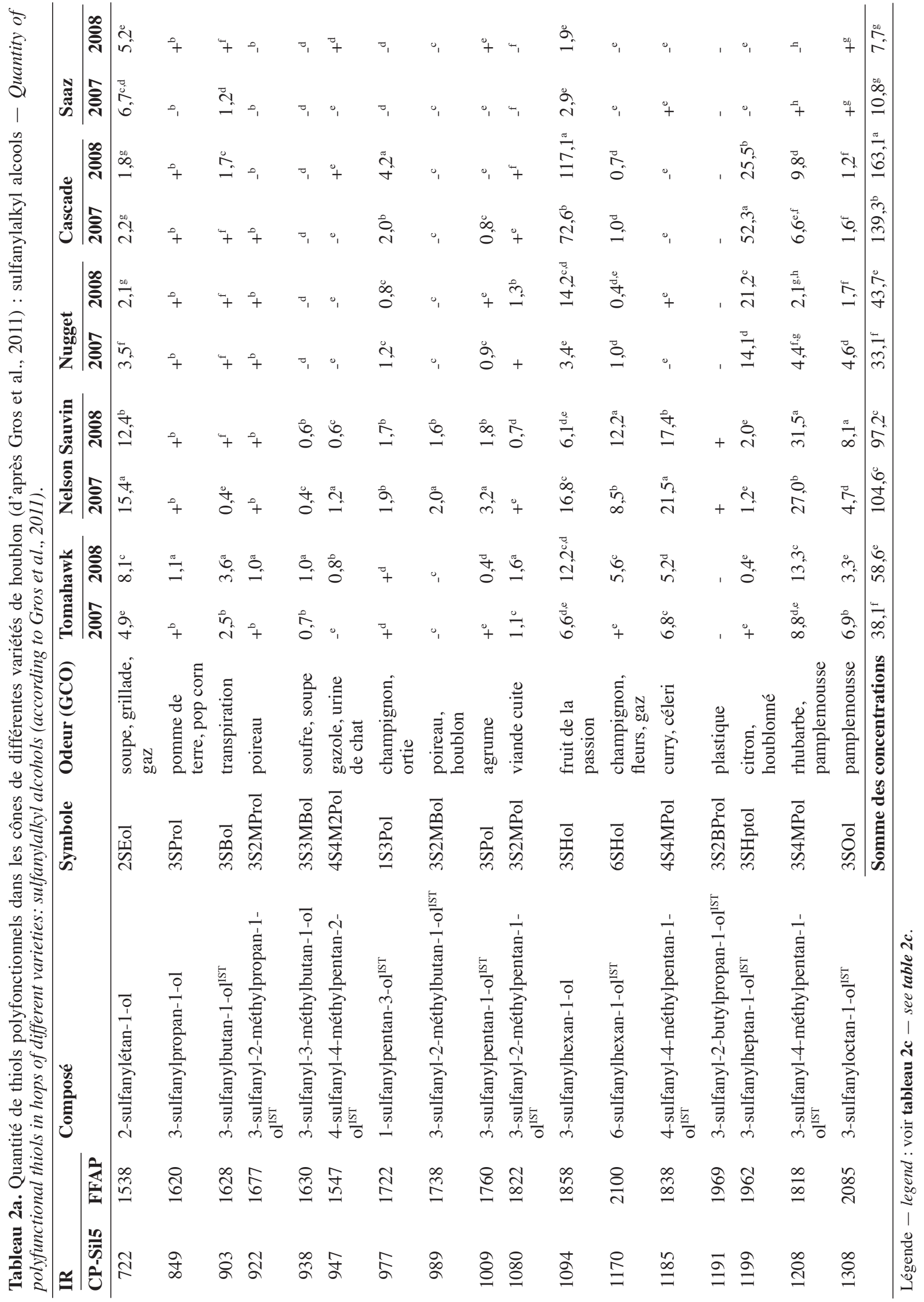




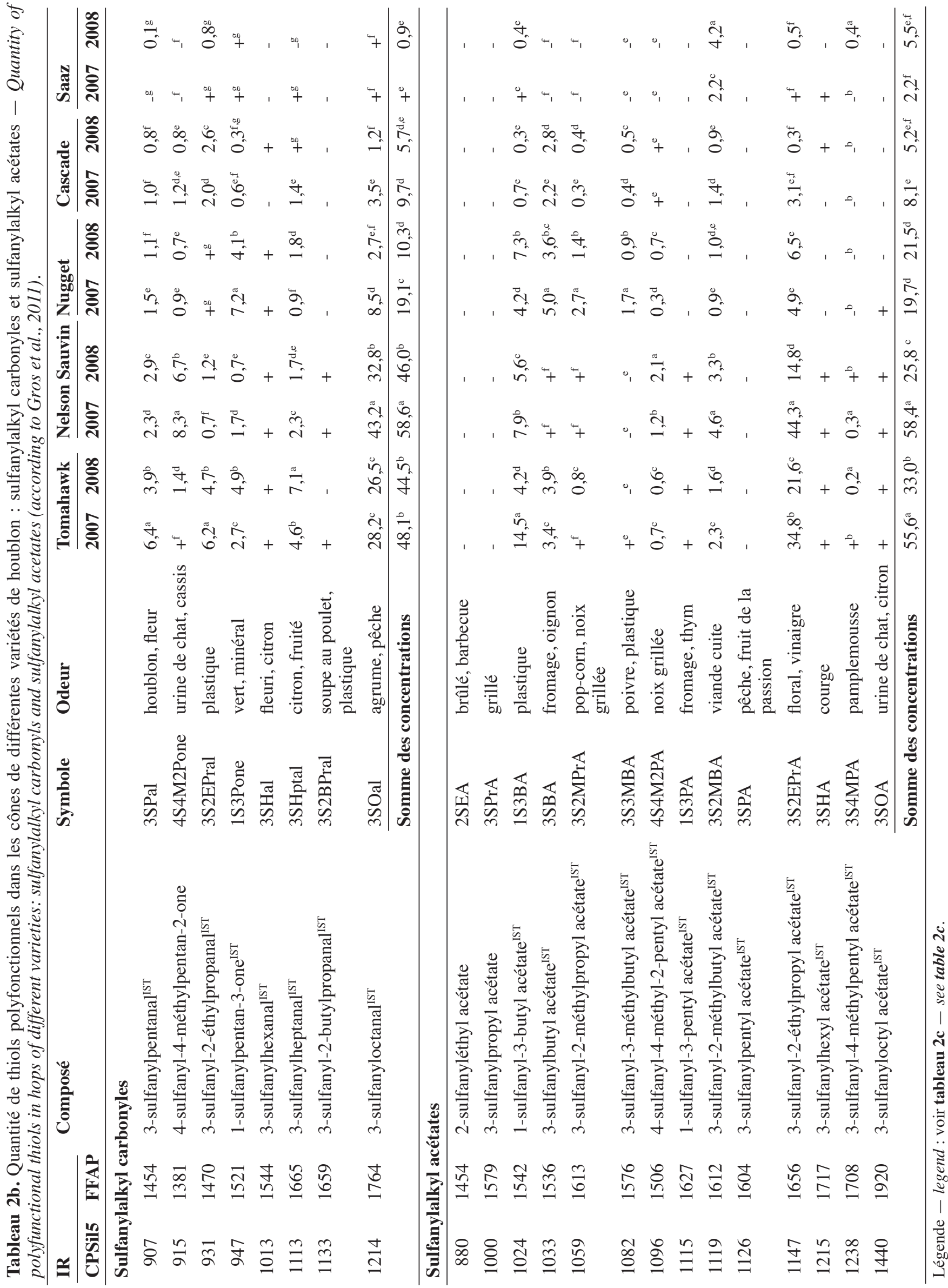




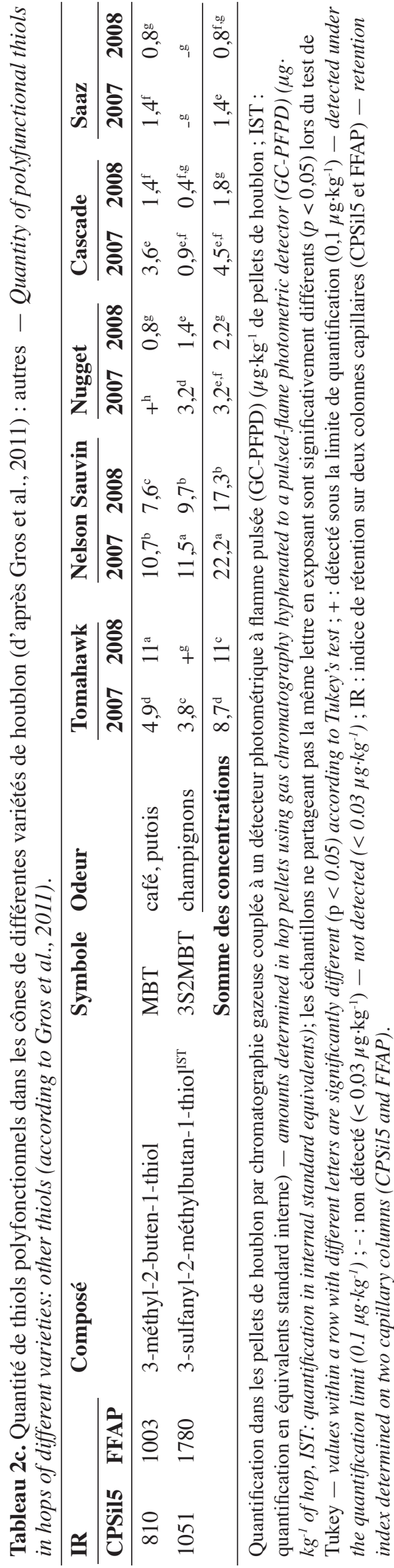

bière, la concentration en 3 SHol peut atteindre plus

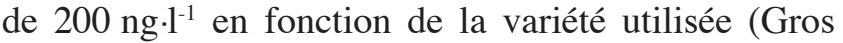
et al., 2011). Dans une étude visant à suivre les concentrations en $3 \mathrm{SHol}$ durant la production de bières avec différents cultivars de houblon, Kishimoto et al. (2008b) ont observé l'absence de 3SHol dans un mout non houblonné mais sa présence dans une bière non houblonnée (20 ng. $\mathrm{l}^{-1}$, Figure 2). On peut dès lors considérer que le malt est une source additionnelle de précurseurs du 3SHol. Durant l'ébullition et la fermentation du mout houblonné, il a été observé que la concentration en $3 \mathrm{SHol}$ commence par augmenter, puis elle tend à se stabiliser. Ce profil peut être expliqué par la libération graduelle du $3 \mathrm{SHol}$ au départ de ses précurseurs (Figure 3). L'adduit cystéiné du 3SHol a été identifié pour la première fois par HPLC/MS/MS dans un houblon de la variété Cascade (Gros et al., 2012). Des teneurs de 261 à $1641 \mu \mathrm{g} \cdot \mathrm{kg}^{-1}$ en 3SHol libérés des précurseurs sont par ailleurs quantifiées après incubation en présence d'apotryptophanase dans les variétés Saaz, Tomahawk, Nelson Sauvin et Cascade (Gros et al., 2013d). Aucune publication ne mentionne à ce jour la présence d'adduits glutathionés dans le houblon.

Le 3-sulfanyl-4-méthylpentan-1-ol (3S4MPol ; Figure 1(26)) à l'arôme d'agrume a été proposé comme un marqueur de la variété Nelson Sauvin, ses concentrations y étant de l'ordre de $32 \mu \mathrm{g} \cdot \mathrm{kg}^{-1}$, alors qu'elles ne dépassent pas $13 \mu \mathrm{g} \cdot \mathrm{kg}^{-1}$ dans les variétés Tomahawk et Cascade $\left(10-13 \mu \mathrm{g} \cdot \mathrm{kg}^{-1}\right)$ (Takoi et al., 2009 ; Gros et al., 2011 ; Gros et al., 2012).

Le 3S3MBol est retrouvé à la fois dans le cône de houblon $\left(0,4-1 \mu \mathrm{g} \cdot \mathrm{kg}^{-1}\right)$ et dans la bière $(0,09-0,58 \mu \mathrm{g}$. $\mathrm{1}^{-1}$ ) où il n'atteint toutefois pas son seuil de perception (1,5 $\mu \mathrm{g} \cdot l^{-1}$; Gros et al., 2011 ; Tran et al., 2015).

Le tableau 2a présente les teneurs retrouvées dans les cônes de houblon et les odeurs correspondantes d'autres sulfanylalkyl alcools dont les 2-sulfanyléthan-1-ol, 3-sulfanylpropan-1-ol, 3-sulfanylbutan-1-ol, 3-sulfanyl-2-méthylpropan-1-ol, 4-sulfanyl-4-méthylpentan-2-ol, 1-sulfanylpentan-3-ol, 3-sulfanyl-2-méthylbutan-1-ol, 3-sulfanylpentan-1-ol, 3-sulfanyl-2-méthylpentan-1-ol, 6-sulfanylhexan-1-ol, 4-sulfanyl-4-méthylpentan-1-ol, 3-sulfanyl-2-butylpropan-1-ol, 3-sulfanylheptan-1-ol et 3-sulfanyloctan$1-\mathrm{ol}$.

\subsection{Les sulfanylalkyl carbonyles}

La 4-sulfanyl-4-méthylpentan-2-one (4S4M2Pone ; arôme de cassis; Figure 1(29)) est probablement l'arôme le plus caractéristique d'un vin de Sauvignon Blanc (Darriet et al., 1995). Comme le 3SHol, le $3 \mathrm{~S} 3 \mathrm{MBol}$ et le 4S4M2Pol, la 4S4M2Pone du vin est générée en grande partie au cours de la fermentation alcoolique (Tominaga et al., 1998a ; Tominaga et al., 


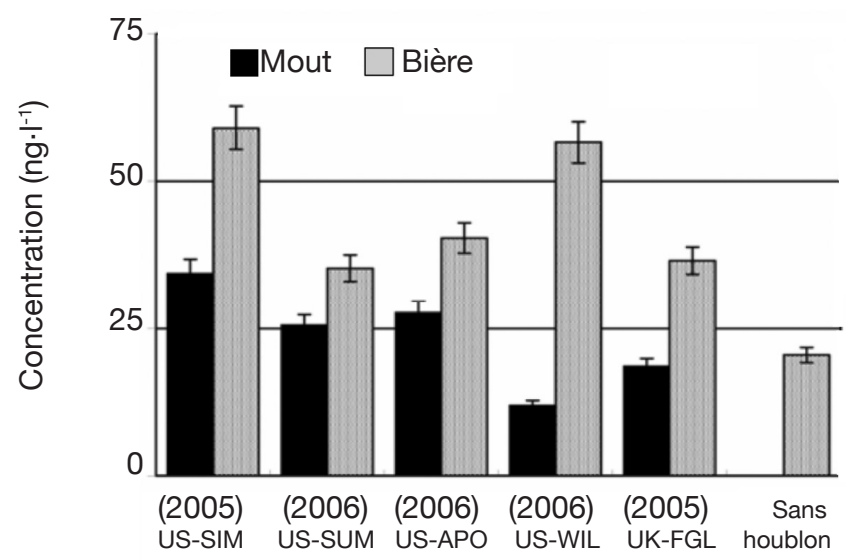

Figure 2. Concentrations $\left(\mathrm{ng} \cdot \mathrm{l}^{-1}\right)$ en $3 \mathrm{SHol}$ dans des mouts bouillis avec différents cônes de houblon (Simcoe [SIM], Summit [SUM], Apollo [APO], Willamet [WIL], Fuggle [FGL]) et les bières correspondantes (d'après Kishimoto et al., 2008b) - Concentrations $\left(n g \cdot l^{-1}\right)$ of $3 \mathrm{SHol}$ in worts boiled with different hop cultivars (Simcoe [SIM], Summit [SUM], Apollo [APO], Willamet [WIL], Fuggle [FGL]) and the corresponding beers (according to Kishimoto et al., 2008b).

1998b). Sa concentration peut atteindre $73 \mathrm{ng} \cdot \mathrm{l}^{-1}$ dans le vin (Tableau 1 ; Tominaga et al., 2000). Ses précurseurs cystéiné et glutathioné ont été retrouvés respectivement à des teneurs de $4 \mu \mathrm{g} \cdot 1^{-1}$ et $1 \mu \mathrm{g} \cdot 1^{-1}$ dans un mout de cépage Sauvignon Blanc (Roland et al., 2010).

Plusieurs sulfanylalkyl carbonyles ont été identifiés et quantifiés dans les cônes de houblon. La somme des concentrations peut y atteindre $59 \mu \mathrm{g} \cdot \mathrm{kg}^{-1}$ (Tableau 2b ; Gros et al., 2011).

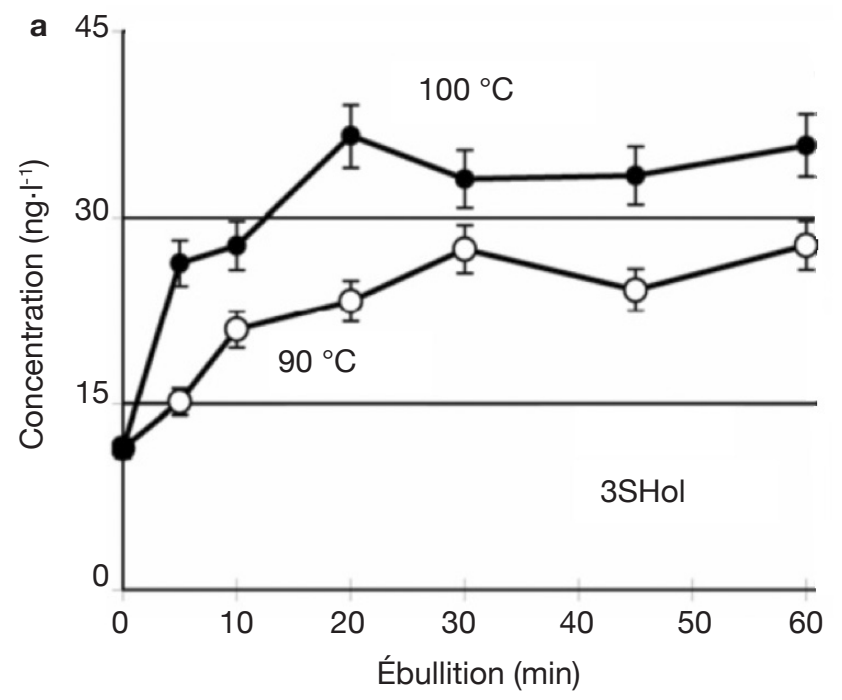

La 4S4M2Pone a d'abord été mise en évidence dans la variété Cascade (Steinhaus et al., 2007) ; elle serait responsable des notes de type «muscat» des bières dérivées. Une étude portant sur un plus grand nombre de variétés a révélé des disparités intervariétales importantes, les teneurs maximales étant retrouvées dans les variétés les plus amères $\left(111 \mu \mathrm{g} \cdot \mathrm{kg}^{-1}\right.$ pour la variété amérisante Simcoe contre $<1 \mu \mathrm{g} \cdot \mathrm{kg}^{-1}$ dans la variété aromatique Saaz) (Figure 4a ; Kishimoto et al., 2008a). Gros et al. (2011) relèvent également des teneurs très élevées $\left(6,7\right.$ à $\left.8,3 \mu \mathrm{g} \cdot \mathrm{kg}^{-1}\right)$ dans la variété Nelson Sauvin. En dosant les ions métalliques divalents dans les mêmes variétés, Kishimoto et al. (2008a) ont montré que les variétés européennes les plus riches en ion cuivrique (sulfate de cuivre non autorisé aux USA) présentent les teneurs les plus basses en 4S4M2Pone (Figure 4b). Au vu des propriétés des mercaptans, une première théorie inspirée des travaux de Darriet et al. (2001) sur le vin attribue ces différences non pas à la variété, mais aux pulvérisations de fongicides à base de sulfate de cuivre (Kishimoto et al., 2008a).

Des teneurs plus élevées en 3-sulfanyloctanal (3SOal ; agrume, pêche ; Figure 1(35)) sont relevées dans la variété Nelson Sauvin (Tableau 2b; Gros et al., 2011). Un profil particulièrement riche en sulfanylalkyl aldéhydes se dégage également pour la variété Tomahawk, avec une présence accrue de 3-sulfanyl-2-éthylpropanal (note soufrée, de plastique ; Figure 1(30)), de 3-sulfanylpentanal (note fleurie; Figure 1(38)), de 3-sulfanylheptanal (note d'agrume ; Figure 1(33)) et de $3 \mathrm{SOal}$.

Une étude réalisée sur 33 variétés selon 11 attributs olfactifs a permis de procéder à un classement sensoriel des houblons. Ainsi, les variétés Nelson Sauvin,

b

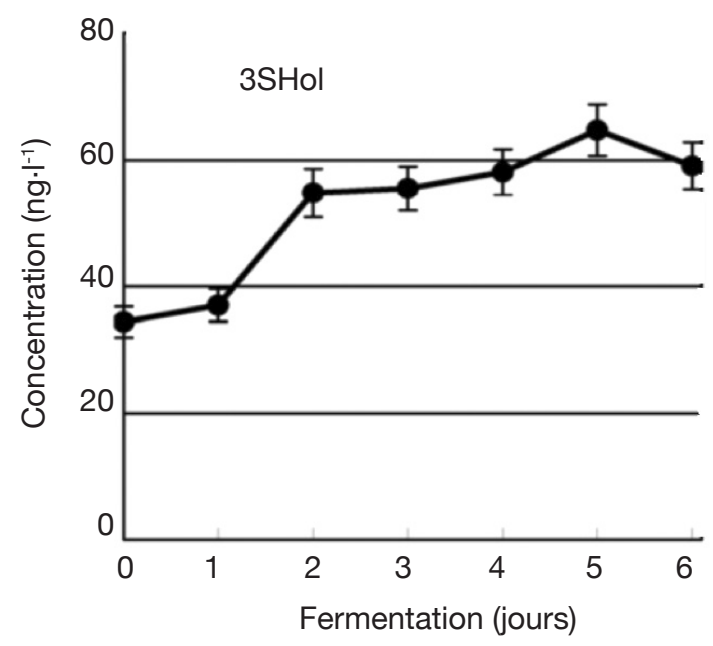

Figure 3. Évolution de la concentration $\left(\mathrm{ng} \cdot \mathrm{l}^{-1}\right)$ en 3-sulfanylhexan-1-ol (3SHol) durant (a) l'ébullition et (b) la fermentation d'un mout houblonné avec la variété Simcoe (d'après Kishimoto et al., 2008b) - Concentration (ng. $\left.l^{-1}\right)$ of 3-sulfanylhexan1-ol (3SHol) during (a) boiling and (b) fermentation of a wort hopped with Simcoe (according to Kishimoto et al., 2008b). 

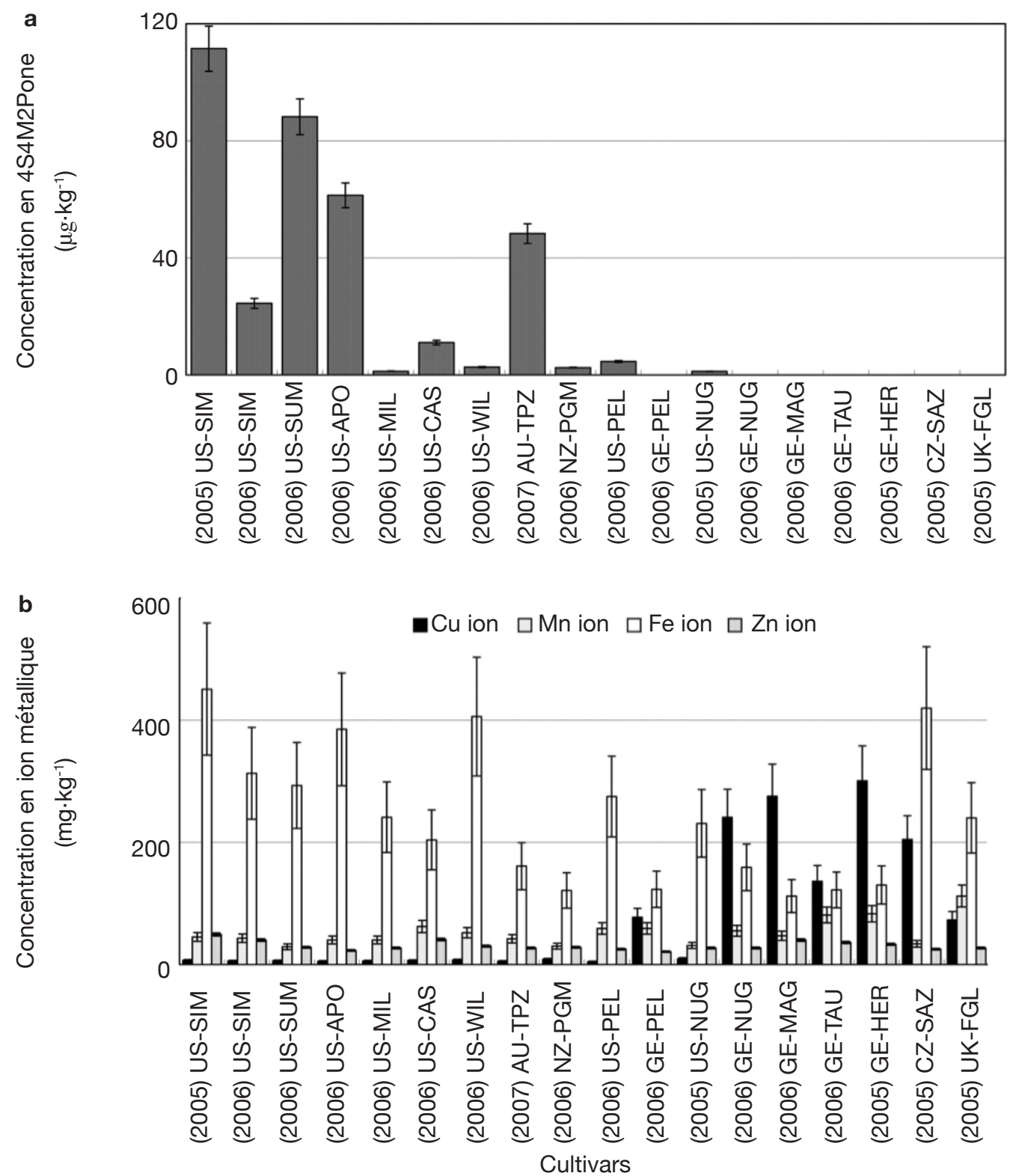

Figure 4. (a) Teneurs en 4-sulfanyl-4-méthylpentan-2-one (4S4M2Pone, $\left.\mu \mathrm{g} \cdot \mathrm{kg}^{-1}\right)$ et (b) teneurs en ions divalents dans les pellets $\left(\mathrm{mg} \cdot \mathrm{kg}^{-1}\right)$ de différents cultivars de houblons récoltés entre 2005 et 2007 aux États-Unis (US), en Australie (AU), en Nouvelle-Zélande (NZ), en Allemagne (GE), en République Tchèque (CZ) ou au Royaume-Uni (UK) (d'après Kishimoto et al., 2008a) - (a) Concentrations of 4-sulfanyl-4-methylpentan-2-one (4S4M2Pone, $\left.\mu \mathrm{g} \cdot \mathrm{kg}^{-1}\right)$ and (b) divalent ion contents in the pellets $\left(\mathrm{mg} \cdot \mathrm{kg}^{-1}\right)$ of different hop cultivars harvested between 2005 and 2007 in the United States (US), Australia (AU), New Zealand (NZ), Germany (GE), Czech Republic (CZ) or United Kingdom (UK) (according to Kishimoto et al., 2008a).

SIM : Simcoe ; SUM : Summit ; APO : Apollo ; MIL : Millenium ; CAS : Cascade ; WIL : Willamette ; TPZ : Topaz ; PGM : Pacific Gem ; PEL : Perle ; NUG : Nugget ; MAG : Magnum ; TAU : Taurus ; HER : Hersbrücker ; SAZ : Saaz ; FGL : Fuggle.

Cascade, Simcoe et Summit riches en 4S4M2Pone ou 3S4MPol (Kishimoto et al., 2008a ; Takoi et al., 2009) forment un groupe défini par les attributs « fruits tropicaux/fruité/agrumes » (Figure 5; Shellhammer, 2011). À l'opposé, les variétés Saaz, Hallertau Tradition, Hallertau Magnum et Nugget plus pauvres en ces thiols sont regroupées à proximité des attributs «épicé » et «vert». Le CTZ (Columbus/Tomahawk/ Zeus) se retrouve dans un groupe marqué par les descripteurs oignon/ail. Quant aux variétés Amarillo et Citra, on les retrouve très proches du Nelson Sauvin sur le plan olfactif. 


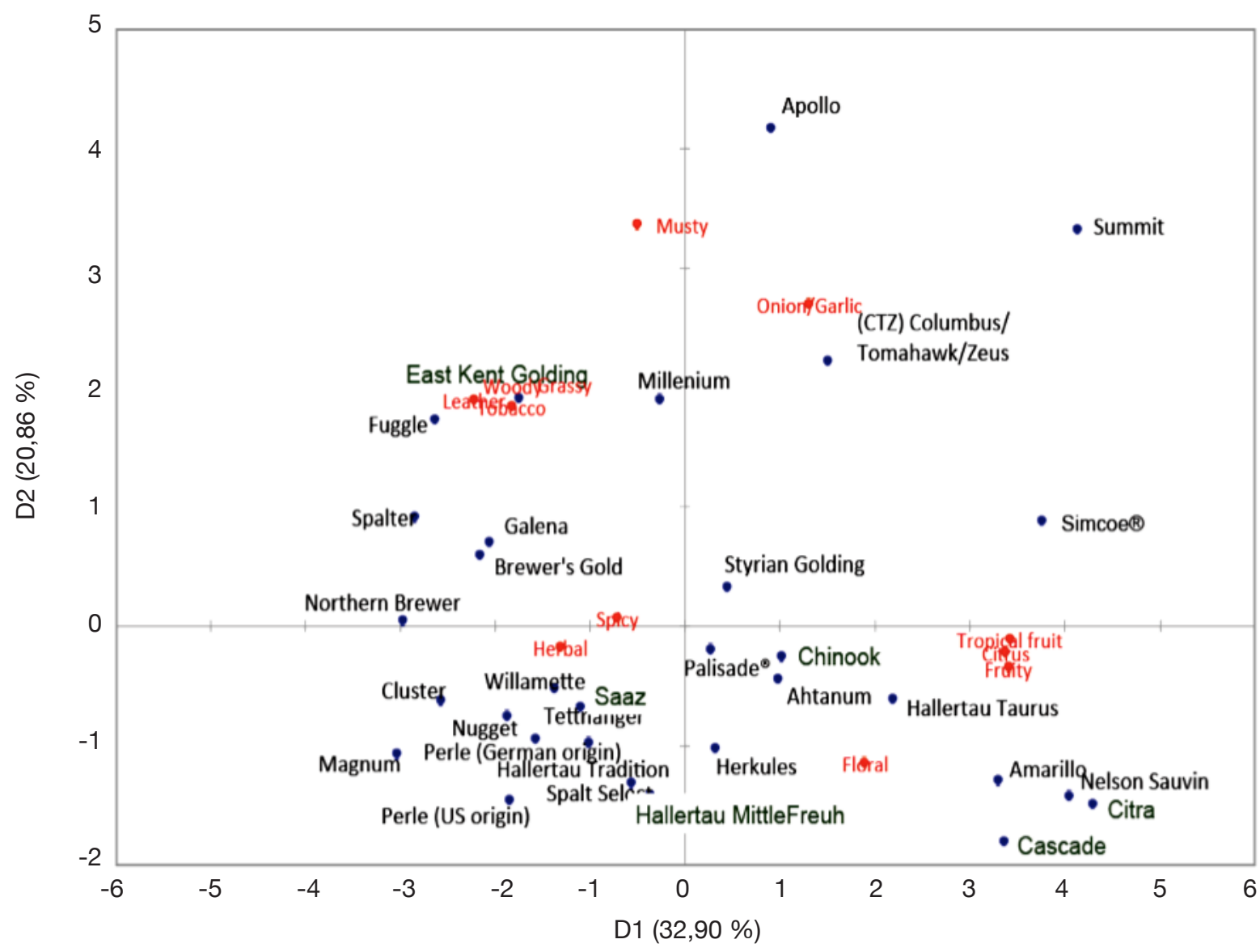

Figure 5. Analyse en composantes principales (D1 : première composante, D2 : deuxième composante) des données sensorielles pour 11 attributs (moisi, cuir, tabac, boisé, herbeux, épicé, oignon/ail, fleuri, fruité, agrumes, fruits tropicaux) et 33 variétés de houblon (d'après Shellhammer et al., 2011) - Principal component analysis (D1: first component, D2: second component) of 11 sensorial attributes (musty, leather, tobacco, woody, grassy, spicy, onion / garlic, flowery, floral, citrus, tropical fruits) and 33 hop varieties (according to Shellhammer et al., 2011).

\subsection{Les sulfanylalkyl acétates}

Aucun sulfanylalkyl acétate n'a été identifié dans le raisin à ce jour. Les deux énantiomères du 3-sulfanylhexyl acétate (3SHA ; Figure 1(11)) ont été retrouvés dans le vin et proviennent de l'acétylation du 3-sulfanylhexan-1-ol par la levure au cours de la fermentation alcoolique (Swiegers et al., 2005 ; Swiegers et al., 2006). L'énantiomère R possède un arôme de fruit de la passion avec un seuil de perception en solution hydroalcoolique de $9 \mathrm{ng} \cdot \mathrm{l}^{-1}$. L'arôme de l'énantiomère $S$ est plus herbacé, avec des notes de buis et un seuil de perception de $2,5 \mathrm{ng} \cdot \mathrm{l}^{-1}$ (Tominaga et al., 2006).

Les sulfanylalkyl acétates sont par contre bien représentés dans les cônes de houblon, leur teneur cumulée pouvant atteindre $58 \mu \mathrm{g} \cdot \mathrm{kg}^{-1}$ (Tableau 2c ; Gros et al., 2011). Treize structures ont ainsi été identifiées, les concentrations les plus élevées étant atteintes pour le 3-sulfanyl-2-éthyl-propyl acétate
(3S2EPrA; Figure 1(10); odeur florale ; 15-44 $\mu \mathrm{g}$. $\mathrm{kg}^{-1}$ dans le Nelson Sauvin et le Tomahawk), le 1-sulfanyl-3-butyl acétate (1S3BA; Figure 1(2); odeur de plastique ; $14,5 \mu \mathrm{g} \cdot \mathrm{kg}^{-1}$ dans le Tomahawk), le 3-sulfanylbutyl acétate (3SBA; Figure 1(3); odeur de fromage; jusque $5 \mu \mathrm{g} \cdot \mathrm{kg}^{-1}$ dans le Nugget) et le 3-sulfanyl-2-méthylbutyl acétate (3S2MBA; Figure 1(8); odeur de viande cuite ; jusque 4,6 $\mu \mathrm{g}$. $\mathrm{kg}^{-1}$ dans le Nelson Sauvin). Des sulfanylalkyl acétates peuvent encore ensuite, comme dans le vin, être créés durant la fermentation brassicole. Ainsi, le 2-sulfanyléthyl acétate (2SEA ; Figure 1(36)), souvent présent à l'état de traces dans les cônes de houblon (Gros et al., 2011), est un thiol polyfonctionnel majoritaire de la bière. De même, la levure convertit le 3SHol en 3SHA grâce à l'alcool acétyl transférase (Swiegers et al., 2005 ; Figure 6). Le seuil de perception du 3SHA dans la bière $\left(5 \mathrm{ng} \cdot \mathrm{l}^{-1}\right)$ étant plus faible que celui du 3 SHol (55 ng.1 $\left.1^{-1}\right)$, il est intéressant de sélectionner une levure efficace dans l'acétylation du $3 \mathrm{SHol}$ pour 

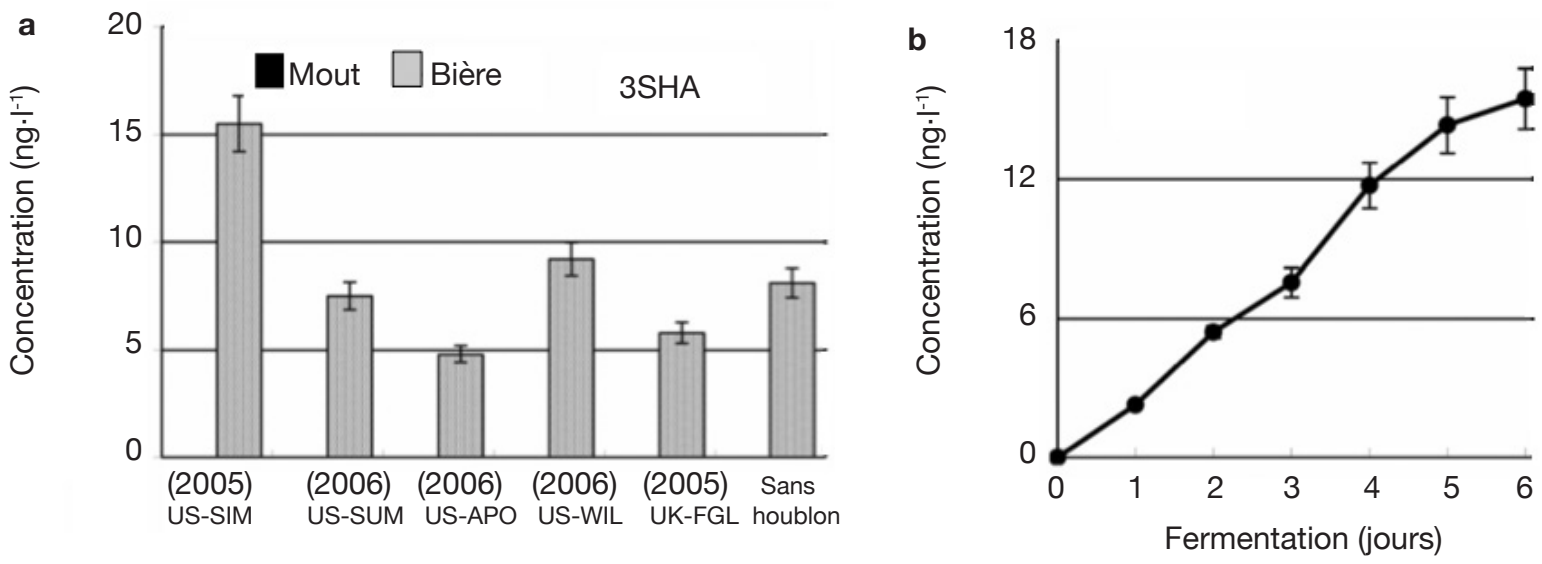

Figure 6. (a) Concentrations (ng. $1^{-1}$ ) en 3-sulfanyl hexylacétate (3SHA) dans des mouts bouillis avec différents cônes de houblons ainsi que dans les bières correspondantes et (b) évolution de la concentration (ng. $\left.1^{-1}\right)$ en 3SHA durant la fermentation d'un mout houblonné avec la variété Simcoe (d'après Kishimoto et al., 2008b) - (a) Concentrations (ng. $l^{-1}$ ) of 3-sulfanyl hexylacetate (3SHA) in worts boiled with hop cones of different cultivars and in the corresponding beers and (b) concentration $\left(n g \cdot l^{-1}\right)$ of 3 SHA during the fermentation of a wort hopped with the Simcoe variety (Kishimoto et al., 2008b).

Abréviations - abbreviations : voir figure 4 - see figure 4.

renforcer l'impact aromatique du houblon (Kishimoto et al., 2008b). Le 3-sulfanyl-4-méthylpentyl acétate (3S4MPA; arôme de pamplemousse ; Figure 1(12)), détecté uniquement dans les cônes de houblons Nelson Sauvin et Tomahawk à l'état de traces $\left(<1 \mu \mathrm{g} \cdot \mathrm{kg}^{-1}\right)$, se retrouve dans les bières correspondantes jusqu'à 96 ng. $\mathrm{l}^{-1}$ (Tableau 2c ; Tableau 3 ; Takoi et al., 2009 ; Gros et al., 2011).

\subsection{Autres thiols polyfonctionnels}

Des traces (jusque $10 \mu \mathrm{g} \cdot \mathrm{kg}^{-1}$ ) de 3-méthyl-2-butèn-1thiol (MBT; Figure 1(37)) ont été retrouvées dans le cône de houblon (Gros et al., 2011). Ce composé est connu pour apporter un défaut de « gout de lumière » ou un arôme de putois à la bière qui n'est pas maintenue à l'abri de la lumière. Son seuil de perception est de l'ordre de 1 à $6,5 \mathrm{ng} \cdot \mathrm{l}^{-1}$ (Goldstein et al., 1993 ; Kobayashi et al., 2005 ; Pozdrik et al., 2006). La concentration en MBT peut ainsi atteindre $15 \mathrm{ng} \cdot \mathrm{l}^{-1}$ dans une bière fraiche de type pils (Kobayashi et al., 2005) et $500 \mathrm{ng} \cdot \mathrm{l}^{-1}$ après $300 \mathrm{~h}$ d'exposition à la lumière (Hughes, 2000). Dans une bière de type ale houblonnée avec la variété Tomahawk, même en l'absence de lumière, sa concentration peut atteindre $580 \mathrm{ng} \cdot \mathrm{l}^{-1}$ (Gros et al., 2012).

Le 3-sulfanyl-2-méthylbutan-1-thiol (3S2MBT ; Figure 1(39); odeur de champignon) se retrouve jusqu'à $11,5 \mu \mathrm{g} \cdot \mathrm{kg}^{-1}$ dans le cône de houblon Nelson Sauvin (Gros et al., 2011).

\section{LES THIOPHÈNES ET ÉPISULFURES DE TERPÈNES}

Le 3-méthylthiophène d'origine terpénique et le 3-(4-méthyl-3-pentényl)thiophène, deux 3-alkylthiophènes (Figure 7a-b), ont été retrouvés dans les huiles essentielles de cônes de houblons riches en

Tableau 3. Teneurs en thiols volatils $\left(\mathrm{ng} \cdot \mathrm{l}^{-1}\right)$ quantifiées dans des extraits aqueux de cônes de houblon de différentes variétés - Content of volatile thiols $\left(n g \cdot l^{-1}\right)$ in aqueous extracts of hops of different varieties (Takoi et al., 2009).

\begin{tabular}{llllllll}
\hline Composé & Symbole & HHT & CSA & HHM & HNU & NPH & NS \\
\hline 4-sulfanyl-4-méthylpentan-2-one & 4S4M2Pone & n.d. & n.d. & 1,8 & n.d. & 0,9 & 149 \\
3-sulfanyl-4-méthylpentyl acétate & 3S4MPA & n.d. & n.d. & n.d. & n.d. & n.d. & $\operatorname{tr}$ \\
3-sulfanylpentan-1-ol & 3SPol & n.d. & n.d. & 6,4 & n.d. & n.d. & 125 \\
3-sulfanyl-4-méthylpentan-1-ol & 3S4MPol & n.d. & 1,6 & 20,5 & 7,9 & n.d. & 848 \\
3-sulfanylhexan-1-ol & 3SHol & 6,0 & 2,3 & 10,0 & 3,0 & n.d. & 8,8 \\
\hline
\end{tabular}

HHT : Hallertau Tradition ; CSA : Czech Saaz ; HHM : Hallertau Magnum ; HNU : Hallertau Nugget ; NPH : Pacific Hallertau ; NS : Nelson Sauvin; n.d. : non détecté - undetected; tr détecté sous forme de traces - detected at trace levels. 
<smiles>Cc1ccsc1</smiles>

(a)<smiles>CC(C)=CCCc1ccsc1</smiles>

(b)

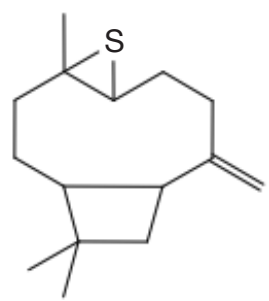

(e)

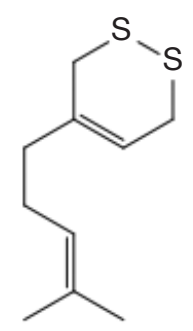

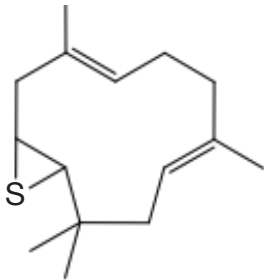

(c)

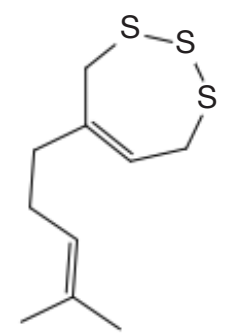

(g)

Figure 7. Structures du (a) 3-méthylthiophène, (b) 3-(4-méthyl-3-pentényl)thiophène, (c) 1,2 épithiohumulène, (d) 4,5-épithiohumulène, (e) 4,5-épithiocaryophyllène, (f) 4-(4-méthylpent-3-enyl)-3,6-dihydro-1,2 -dithiine et (g) -trisulfure - Structure of (a) 3-methylthiophene, (b) 3-(4-methyl-3-pentenyl)thiophene, (c) 1,2 epithiohumulene, (d) 4,5-epithiohumulene, (e) 4,5-epithiocaryophyllene, (f) 4-(4-methyl-3-enyl)-3,6-dihydro-1,2 -dithiine and (g) -trisulfide.

soufre élémentaire résiduel (cultures traitées ; Suggett et al., 1979).

En présence de lumière ou sous l'effet d'un traitement thermique, il a été observé que l'humulène, le caryophyllène et le myrcène peuvent former des épisulfures (Figure 7c-g ; Peppard et al., 1979 ; Seaton etal., 1987). Jusqu'à $9300 \mathrm{mg} \cdot 1^{-1} \mathrm{de} 1,2$ épithiohumulène et $3360 \mathrm{mg} \cdot \mathrm{l}^{-1} \mathrm{de} 4,5$-épithiohumulène ont été retrouvés dans des huiles essentielles de cônes de houblon contenant $5000 \mathrm{mg} \cdot \mathrm{kg}^{-1}$ de soufre élémentaire (cultures traitées ; Peppard et al., 1979). Une dizaine d'adduits soufrés du myrcène ont également été identifiés. Parmi ceux-ci, la 4-(4-méthyl-3-pentényl)-3,6-dihydro-1,2dithiine (Figure 7f) a été caractérisée chimiquement et a été retrouvée jusqu'à $1000 \mathrm{mg} \cdot \mathrm{l}^{-1}$ dans les huiles essentielles de cônes de houblons obtenues après distillation (Seaton et al., 1987).

\section{LES ALKYLSULFURES ET POLYSULFURES}

Les alkylsulfures et polysulfures ont été, à notre connaissance, très peu étudiés dans le raisin. Leur présence est par contre mentionnée dans le vin. Le diméthylsulfure (DMS) a été retrouvé dans les vins de Petit et de Gros Maseng à des teneurs allant de 3 à $711 \mu \mathrm{g} \cdot 1^{-1}$. Son seuil de perception dans le vin varie entre 22 et $60 \mu \mathrm{g} \cdot 1^{-1}$. Le DMS participe aux notes d'asperge et de maïs dans les vins blancs, alors que dans les vins rouges, il intensifie les caractères fruités en association à des notes de truffes. La teneur en DMS du vin augmente au cours du stockage où il est produit chimiquement à partir de précurseurs (Dagan et al., 2006). Bien qu'encore non identifiée dans le vin, la S-méthylméthionine, présente dans le raisin, est un précurseur suspecté pour le DMS (Ségurel et al., 2005 ; Dagan et al., 2006). Durant la fermentation, l'éthanol peut réagir avec 1 ' $\mathrm{H}_{2} \mathrm{~S}$ pour générer de l'éthanethiol (arôme d'oignon). En conditions oxydantes, cet éthanethiol, peut ensuite être oxydé en diéthyldisulfure apportant des arômes désagréables au vin (Ribéreau-Gayon et al., 2006). Les rayons UV peuvent, quant à eux, induire une dégradation photooxidative de la méthionine conduisant à la production de méthanethiol. Celui-ci peut aussi être oxydé pour former cette fois le diméthyldisulfure (Ribéreau-Gayon et al., 2006).

Contrairement au raisin, le cône de houblon renferme les diméthyl -disulfure, -trisulfure et -tétrasulfure (Figure 8a-c; notes de solvant; de verdure et de choux), ainsi que le diéthyldisulfure (note de plastique brûlé) (extraits Likens Nickerson de la variété Challenger; Lermusieau et al., 2001 ; Tableau 4). Le diméthyltrisulfure (DMTS) de la bière fraîche est pourtant principalement issu de la réaction de $l^{\prime} \mathrm{H}_{2} \mathrm{~S}$ de fermentation avec l'acide sulfénique du cône de houblon, lui-même issu de la S-méthylcystéine sulfoxyde (Peppard, 1978; Lermusieau et al., 2003). Dans une bière vieillie, une voie de synthèse supplémentaire au départ de méthionol ou de méthional l'amène souvent au-dessus de son seuil de perception (Gijs et al., 2000a ; Gijs et al., 2002). Le DMTS est totalement absent des bières non houblonnées. Le 3,3-diméthylallylméthyldisulfure (Figure 8d) a été 


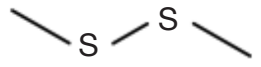

(a)

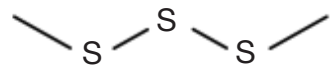

(b)

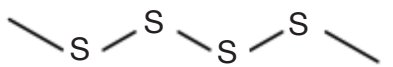

(c)

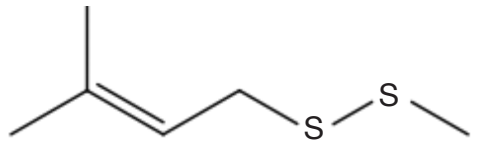

(d)

Figure 8. Structures du (a) diméthyl disulfure, (b) diméthyl trisulfure, (c) diméthyl tétrasulfure, (d) 3,3-diméthylallylméthyl disulfure - Structure of (a) dimethyl disulfide, (b) dimethyl trisulfide, (c) dimethyl tetrasulfide, (d) 3,3- dimethylallylmethyl disulfide.

retrouvé dans des huiles de cônes de houblon traitées thermiquement (Takabe et al., 1970).

\section{LES THIOESTERS}

À notre connaissance, aucun thioester n'a encore été identifié dans le raisin.

Les S-méthylthio -acétate, -butanoate, -isovalérate, -pentanoate, -hexanoate, -heptanoate, -décanoate et -(E)-4-méthyl-3-hexénoate (responsables de notes de légumes cuits, de fromage, d'ananas ou de rance) sont présents dans les huiles essentielles de cônes de houblons (Figure 9a-h ; Tableau 4; Seaton et al., 1981 ; Lermusieau et al., 2001 ; Gijs et al., 2003 ; Miyazato, 2013). La voie de biosynthèse de ces thioesters n'y a toutefois pas été investiguée. À l'instar de la voie de formation des esters en fermentation, nous suspectons dans la plante une thioestérification du méthanethiol (issu de la dégradation de la méthionine) par l'acylCoA(Walker, 1991 ; Lermusieau et al., 2003). L'acétylation du méthanethiol en S-méthylthioacétate<smiles>CCCC(=O)SC</smiles>

(a)<smiles>CSC(=O)CC(C)C</smiles>

(c)

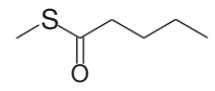

(d)<smiles>CCCCCCCCCC(=O)SCCCCCCC(=O)SC</smiles>

(e)

(f)

(g)<smiles>CC/C(C)=C/CC(=O)SC</smiles>

(h)

Figure 9. Structures des S-méthylthioesters retrouvés dans le cône de houblon - Structures of S-methylthioesters found in hop cones.

S-méthylthio (a) -acétate, (b) -butanoate, (c) -isovalérate, (d) -pentanoate, (e) -hexanoate, (f) -heptanoate, (g) -décanoate, (h) -(E)-4-méthyl-3-hexénoate - S-methylthio (a) -acetate, (b) butanoate, $(\boldsymbol{c})$-isovalerate, $(\boldsymbol{d})$ pentanoate, $(\boldsymbol{e})$-hexanoate, $(\boldsymbol{f})$ -heptanoate, $(\mathrm{g})$-decanoate, $(\boldsymbol{h})$-(E)-4-methyl-3-hexenoate.
Tableau 4. Teneurs $\left(\mathrm{mg} \cdot \mathrm{kg}^{-1}\right)$ en sulfures et polysulfures, et thioesters dans un cône de houblon séché - Sulfides, polysulfides and thioesters contents $\left(\mathrm{mg}^{\mathrm{kg}} \mathrm{kg}^{-1}\right)$ in a dried hop cone.

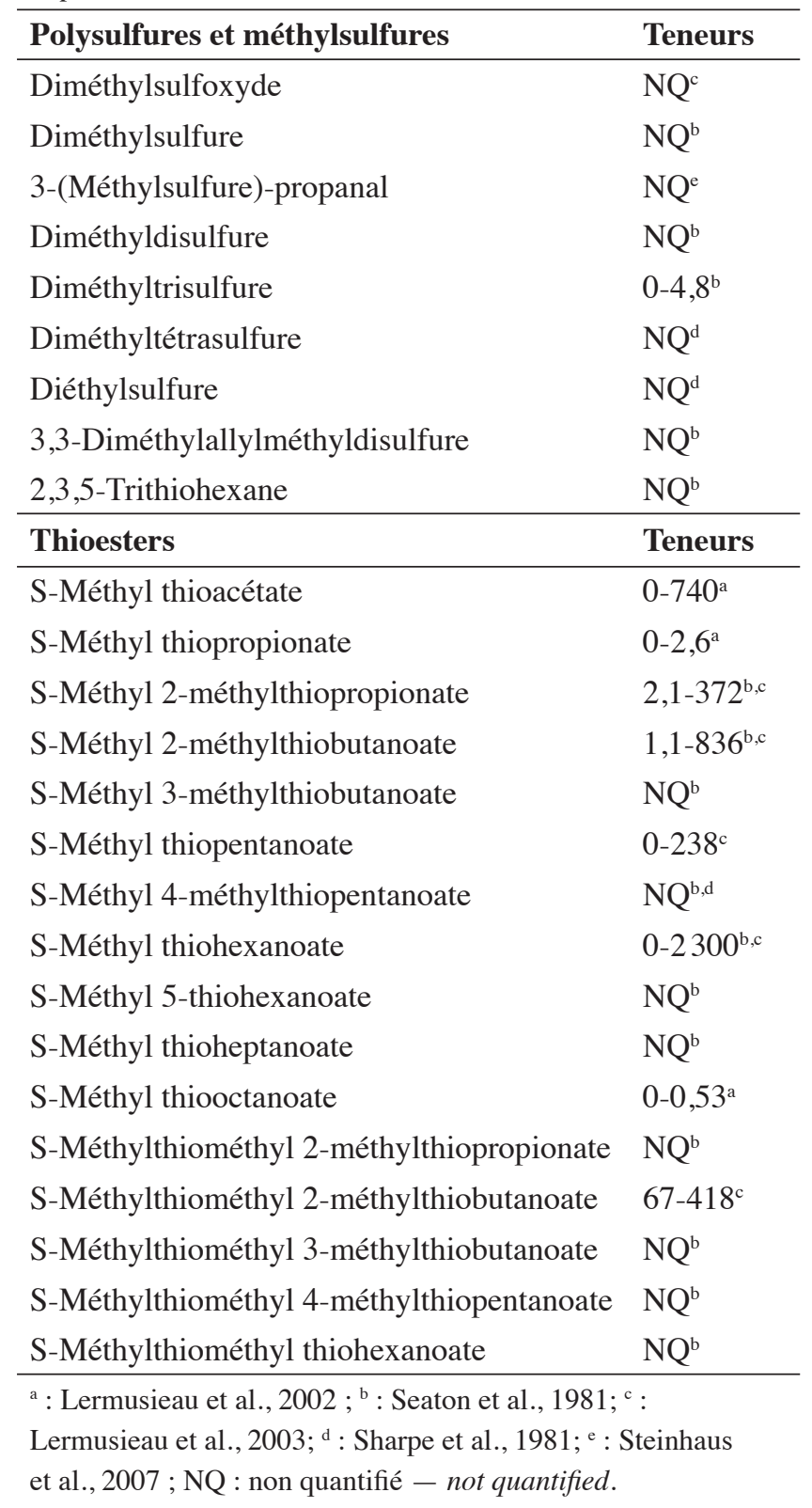


est également observée au cours de la fermentation (Walker, 1991).

Plus que la variété, la région et les conditions de culture du houblon conditionnent les teneurs en ces composés. Les traitements post-récolte ont également un impact. Au cours du séchage, le traitement thermique favorise l'augmentation de la teneur en thioesters (Peppard, 1981). Pour la variété Wye Challenger, une augmentation moyenne de $40 \%$ en thioesters a été observée après le séchage (Seaton et al., 1987). Le moment de l'ajout des cônes de houblon au cours du processus brassicole a une grande influence sur le profil en thioesters de la bière finie (Suggett et al., 1979). Ainsi, le S-méthylthiohexanoate (odeur d'ananas ; seuil de perception dans la bière de $1 \mu \mathrm{g} \cdot \mathrm{l}^{-1}$; Figure 9e) est actif d'un point de vue organoleptique dans une bière houblonnée à cru alors que le S-méthylthiobutanoate (odeur de fromage, seuil de perception dans la bière de $0,01 \mu \mathrm{g} \cdot \cdot^{-1}$; Figure 9b) et le S-méthylthioisovalérate (odeur de fromage; Figure 9c) sont dominants dans les bières houblonnées en cuve d'ébullition (Guadagni et al., 1966 ; Berger et al., 1999 ; Gijs et al., 2000b ; Lermusieau et al., 2001 ; Gijs et al., 2003).

\section{LES PYRAZINES}

Les pyrazines sont responsables soit d'odeurs de type noisette (surtout les alkylpyrazines de faible lipophilie), soit d'arômes de verdure (méthoxypyrazines et pyrazines de plus grande taille) (Hérent et al., 1998). Les 3-isobutyl-2-méthoxypyrazine (IBMP, note de poivron vert, seuil de perception dans le vin de $15 \mathrm{ng} \cdot \mathrm{l}^{-1}$ ) et 3-isopropyl-2-méthoxypyrazine (IPMP, note de petit pois) jouent un rôle important dans les notes herbacées non désirées de vins issus des cépages Cabernet Sauvignon, Merlot, Cabernet Franc et Carménère (Hashizume et al., 1996; Roujou de Boubée et al., 2000 ; Belancic et al., 2007 ; Robinson et al., 2014). Ils contribuent aussi aux arômes végétatifs variétaux des cépages blancs Chardonnay, Sauvignon Blanc, Sémillon et Riesling (Allen et al., 1991 ; Hashizume et al., 1999). Leur biogenèse est associée au métabolisme du glyoxal et des acides aminés : leucine, isoleucine, valine (Murray et al., 1975). Les $O$-méthyltransférases dépendantes de la S-adénosyl-L-méthionine méthylent les 3-alkyl-2-hydroxypyrazines pour la formation des 3-alkyl-2-méthoxypyrazines (Figure 10). Les

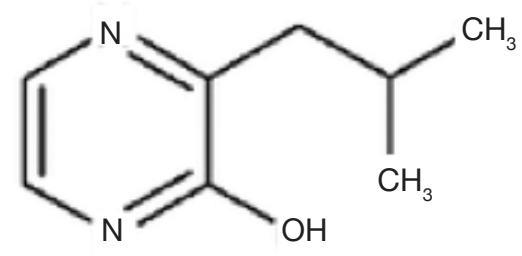

3-isobutyl-2-hydroxypyrazine

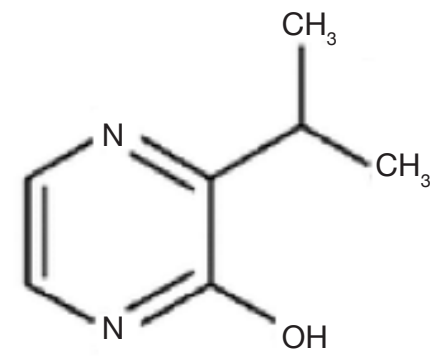

3-isopropyl-2-hydroxypyrazine
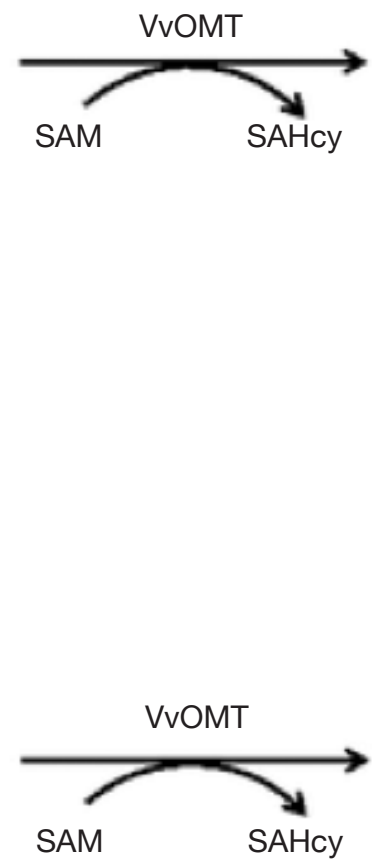

3-isopropyl-2-methoxypyrazine

Figure 10. Voie de synthèse proposée pour les 3-alkyl-2-méthoxypyrazines impliquant la Vitis vinifera $O$-méthyltransférase (VvOMT) en présence de la S-adénosyl-L-méthionine (SAM) comme donneur de méthyle aux 3-alkyl-2-hydroxypyrazines 3-Alkyl-2-methoxypyrazines synthetic pathway involving Vitis vinifera O-methyltransferase (VvOMT) in the presence of S-adenosyl-L-methionine (SAM) as a methyl donor to 3-alkyl-2-hydroxypyrazines (Vallarino et al., 2011). 
gènes VvOMT1 et VvOMT3 qui codent pour les $O$-méthyltransférases ont été identifiés dans les grappes de Cabernet Sauvignon (Dunlevy et al., 2010 ; Vallarino et al., 2011 ; Dunlevy et al., 2013 ; Guillaumie et al., 2013) ; 53 \% d'IBMP s'y retrouvent dans la rafle. Dans la baie, $67 \%$ d'IBMP sont localisés dans la peau pour seulement $1 \%$ dans la chair, le reste se retrouvant dans les pépins (Roujou de Boubée et al., 2002).

La teneur en méthoxypyrazines diminue après la véraison (Roujou de Boubée et al., 2000 ; Guillaumie et al., 2013). L'exposition des baies au soleil amplifie cette dégradation (Hashizume et al., 1999), alors que les climats froids favorisent des teneurs plus élevées (Lacey et al., 1991). Localisées dans la baie et dans la rafle, les méthoxypyrazines voient leur teneur augmenter dans le vin avec le temps de macération (Hashizume et al., 1997 ; Kotseridis et al., 1999). Dans la bière, on retrouve principalement des alkylpyrazines dont la 2,6-diméthylpyrazine, la 2-méthylpyrazine et la 2-éthyl-3-méthylpyrazine, en particulier lorsque l'on utilise des malts spéciaux touraillés à plus hautes températures (Vandecan et al., 2010 ; Riu-Aumatell et al., 2014). Ils sont alors issus des réactions de Maillard, à l'instar de nombreux furannes, thiazoles et thiophènes. Notons toutefois que des traces de méthoxypyrazines peuvent provenir d'orges torréfiées (non germées). À notre connaissance, aucune pyrazine n'a été identifiée dans le cône de houblon.

\section{CONCLUSIONS}

Plusieurs analogies et différences ont été relevées entre le cône de houblon et la baie de raisin. Le 3SHol caractérisé par des arômes de pamplemousse ou de fruit de la passion, ainsi que son précurseur cystéiné, ont été identifiés dans les deux matrices. À l'instar des études menées en œnologie, le précurseur glutathioné devrait maintenant être investigué dans le cône de houblon. Aucun sulfanylalkyl acétate n'a été retrouvé dans le raisin, contrairement au cône de houblon. Cependant, la levure est capable d'estérifier le 3SHol en 3SHA en fermentation grâce à l'alcool acétyl transférase. Les thiophènes, épisulfures, sulfures, polysulfures et thioesters sont principalement retrouvés dans le cône de houblon, alors que les méthoxypyrazines ne sont observées que dans la vigne.

\section{Bibliographie}

Allen M.S., Lacey M.J., Harris R.L.N. \& Brown W.V., 1991. Contribution of methoxypyrazines to Sauvignon Blanc wine aroma. Am. J. Enol. Vitic., 42, 109-112.

Allen T. et al., 2011. Influence of grape-harvesting steps on varietal thiol aromas in Sauvignon Blanc wines. J. Agric. Food Chem., 59, 10641-10650.
Belancic A. \& Agosin E., 2007. Methoxypyrazines in grapes and wines of Vitis vinifera cv. Carmenere. Am. J. Enol. Vitic., 58, 462-469.

Berger C. et al., 1999. Combinatorial approach to flavor analysis. 2. Olfactory investigation of a library of S-methylthioesters and sensory evaluation of selected components. J. Agric. Food Chem., 47, 32743279.

Capone D.L., Sefton M.A., Hayasaka Y. \& Jeffery D.W., 2010. Analysis of precursors to wine odorant 3-mercaptohexan-1-ol using HPLC-MS/MS: resolution and quantitation of diastereomers of 3-S-cysteinylhexan1-ol and 3-S-glutathionylhexan-1-ol. J. Agric. Food Chem., 58, 1390-1395.

Dagan L., 2006. Potentiel aromatique des raisins de Vitis vinifera L. cv. Petit Manseng et Gros Manseng. Contribution à l'arôme des vins de pays Côtes de Gascogne. Thèse de doctorat: École Nationale Supérieure Agronomique de Montpellier (France).

Darriet P. et al., 1995. Identification of a powerful aromatic component of Vitis vinifera L. var. Sauvignon wines: 4-mercapto-4-methylpentan-2-one. Flavour Fragance. J., 10, 385-392.

Darriet P. et al., 2001. Effects of copper fungicide spraying on volatile thiols of the varietal aroma of Sauvignon Blanc, Cabernet Sauvignon and Merlot wines. Vitis, 40, 93-99.

Dunlevy J.D. et al., 2010. Two O-methyltransferases involved in the biosynthesis of methoxypyrazines: Grape-derived aroma compounds important to wine flavour. Plant Mol. Biol., 74, 77-89.

Dunlevy J.D. et al., 2013. A methyltransferase essential for the methoxypyrazine-derived flavour of wine. Plant J., 75, 606-617.

Gijs L., Perpète P., Timmermans A. \& Collin S., 2000a. 3-Methylthiopropionaldehyde as precursor of dimethyltrisulfide in aged beers. J. Agric. Food Chem., 48, 6196-6199.

Gijs L. et al., 2000b. Retention of sulfur flavours by food matrix and determination of sensorial data independent of the medium composition. J. Agric. Food Chem., 69, 319-330.

Gijs L., Chevance F., Jerkovic V. \& Collin S., 2002. How low $\mathrm{pH}$ can intensify beta-damascenone and dimethyl trisulfide production through beer aging. J. Agric. Food Chem., 50, 5612-5616.

Gijs L., Vermeulen C. \& Collin S.,2003. Review. Occurence et voies de formation des arômes soufrés dans la bière. 1. Les sulfures et les polysulfures. Cerevisia, 28, 37-46.

Goldstein H., Rader S. \& Murakami A., 1993. Determination of 3-methyl-2-butene-1-thiol in beer. J. Am. Soc. Brew. Chem., 51, 70-74.

Gros J., Nizet S. \& Collin S., 2011. Occurrence of odorant polyfunctional thiols in the super alpha Tomahawk hop cultivar. Comparison with the thiol-rich Nelson Sauvin bitter variety. J. Agric. Food Chem., 59, 8853-8865. 
Gros J., Peeters F. \& Collin S., 2012. Occurrence of odorant polyfunctional thiols in beers hopped with different cultivars. First evidence of an S-cysteine conjugate in hop (Humulus lupulus L.). J. Agric. Food Chem., 60, 7805-7816.

Gros J., Kankolongo C.M.L. \& Collin S., 2013a. Revue sur les étonnantes analogies et les différences relevées entre un cône de houblon et une baie de raisin. Partie I : pratiques culturales, anatomie et sélection de nouvelles variétés. Cerevisia, 38, 61-70.

Gros J., Kankolongo C.M.L. \& Collin S., 2013b. Revue sur les étonnantes analogies et les différences relevées entre un cône de houblon et une baie de raisin. Partie II : les constituants majeurs. Cerevisia, 38, 79-88.

Gros J., Tran T.T.H. \& Collin S., 2013c. Revue bibliographique sur les adduits cystéinés et glutathionés de la vigne en vue de leur investigation dans le houblon et la bière. Cerevisia, 38, 3-14.

Gros J., Tran T.T.H. \& Collin S., 2013d. Enzymatic release of odourant polyfunctional thiols from cysteine conjugates in hop. J. Inst. Brew., 119, 221-227.

Guadagni D.G., Buttery R.G. \& Harris J., 1966. Odour intensities of hop oil components. J. Sci. Food Agric., 17, 142-144.

Guillaumie S. et al., 2013. Genetic analysis of the biosynthesis of 2-methoxy-3-isobutylpyrazine, a major grape-derived aroma compound impacting wine quality. Plant Physiol., 162, 604-615.

Hashizume K.\& Umeda N., 1996. Methoxypyrazine content of Japanese red wines. Biosci. Biotechnol. Biochem., 60 , 802-805.

Hashizume K. \& Samuta T., 1997. Green odorants of grape cluster stem and their ability to cause a wine stemmy flavor. J. Agric. Food Chem., 45, 1333-1337.

Hashizume K. \& Samuta T., 1999. Grape maturity and light exposure affect berry methoxypyrazine concentration. Am. J. Enol. Vitic., 50, 194-198.

Hérent M.F. \& Collin S., 1998. Pyrazine and thiazole structural properties and their influence on the recovery of such derivatives in aroma extraction procedures. J. Agric. Food Chem., 46, 1975-1980.

Hughes P., 2000. Reducing power and sulfur compounds. In: Conference Chair de Clerck IX, Louvain-la-Neuve, Belgium.

Kankolongo C.M.L., Gros J. \& Collin S., 2014. Revue sur les étonnantes analogies et les différences relevées entre un cône de houblon et une baie de raisin. Partie III : arômes - terpènes, C13-norisoprénoïdes, hydrocarbures et dérivés oxygénés non terpéniques. Cerevisia,38, 103-117.

Kishimoto T. et al., 2008a. Comparison of 4-mercapto4-methylpentan-2-one contents in hop cultivars from different growing regions. J. Agric. Food Chem., 56, 1051-1057.

Kishimoto T. et al., 2008b. Behaviors of 3-mercaptohexan1-ol and 3-mercaptohexyl acetate during brewing processes. J. Am. Soc. Brew. Chem., 66, 192-196.
Kobayashi M., Iida A., Kagami N. \& Kawature K., 2005. Formation and control of 3-methyl-2-butene-1-thiol during fermentation. In: Proceedings of the $30^{\text {th }}$ Congress of the European Brewery Convention, Prague, May 2005, 333-339.

Kotseridis Y. et al., 1999. Effects of selected viticultural and enological factors on levels of 2-methoxy-3isobutylpyrazine in wines. J. Int. Sci. Vigne Vin, 33, 19-23.

Lacey M.J., Allen M.S., Harris R.L.N. \& Brown W.V., 1991. Methoxypyrazines in Sauvignon Blanc grapes and wines. Am. J. Enol. Vitic., 42, 103-108.

Lermusieau G., Bulens M. \& Collin S., 2001. Use of GColfactometry to identify the hop aromatic compounds in beer. J. Agric. Food Chem., 49, 3867-3874.

Lermusieau G. \& Collin S., 2002. Hop aroma extraction and analysis. In: Jackson J.F. \& Linskens H.F. Analysis of taste and aroma. Berlin/Heidelberg, Germany: SpringerVerlag, 69-88.

Lermusieau G. \& Collin S., 2003. Volatile sulfur compounds in hops and residual concentrations in beer - a review. J. Am. Soc. Brew. Chem., 61, 109-113.

Miyazato H., 2013. Identification of the compounds responsible for the sweat-like odor in hop (Humulus lupulus L.) volatile oil. J. Food Res., 2, 34-47.

Murat M.L., Tominaga T. \& Dubourdieu D., 2001. Assessing the aromatic potential of Cabernet Sauvignon and Merlot musts used to produce rose wine by assaying the cysteinylated precursor of 3-mercaptohexan-1-ol. J. Agric. Food Chem., 49, 5412-5417.

Murray K.E. \& Whitfield F.B., 1975. The occurrence of 3-alkyl-2-methoxypyrazines in raw vegetables. J. Sci. Food Agric., 26, 973-986.

Peppard T.L., 1978. Dimethyltrisulfide, its mechanism of formation in hop oil and effect on beer flavor. J. Inst. Brew., 84, 337-340.

Peppard T.L., 1981. Volatile organosulphur compounds in hops and hop oils: a review. J. Inst. Brew., 87, 376-385.

Peppard T.L. \& Laws D.R.J., 1979. Hop derived sulphur compounds and their effect on beer flavour. In: Proceedings of the $17^{\text {th }}$ Congress of the European Brewery Convention, Berlin West, Germany, December 1979, 91-104.

Pozdrik R., Roddick F., Rogers P. \& Nguyen T., 2006. Spectrophotometric method for exploring 3-methyl-2butene-1-thiol (MBT) formation in lager. J. Agric. Food Chem., 54, 6123-6129.

Ribéreau-Gayon P.,Glories Y.,Maujean A.\&Dubourdieu D., 2006. Handbook of enology. The chemistry of wine stabilisation and treatments. Vol.2. Wiley.

Riu-Aumatell M. et al., 2014. Assessment of the aroma profiles of low-alcohol beers using HS-SPME-GC-MS. Food Res. Int., 57, 196-202.

Robinson A.L. et al., 2014. Origins of grape and wine aroma. Part 1. Chemical components and viticultural impacts. Am. J. Enol. Vitic., 65, 1-24. 
Roland A. et al., 2010. Validation of a nanoliquid chromatography-tandem mass spectrometry method for the identification and the accurate quantification by isotopic dilution of glutathionylated and cysteinylated precursors of 3-mercaptohexan-1-ol and 4-mercapto-4methylpentan-2-one in white grape juices. J.Chromatogr. A., 1217, 1626-1635.

Roland A. et al., 2011. Distribution of varietal thiol precursors in the skin and the pulp of Melon B. and Sauvignon Blanc grapes. Food Chem., 125, 139-144.

Roujou de Boubée D., Van Leeuwen C. \& Dubourdieu D., 2000. Organoleptic impact of 2-methoxy-3isobutylpyrazine on red Bordeaux and Loire wines. Effect of environmental conditions on concentrations in grapes during ripening. J. Agric. Food Chem ., 48, 48304834.

Roujou de Boubée D., Cumsille A.M., Pons M. \& Dubourdieu D., 2002. Location of 2-methoxy-3isobutylpyrazine in Cabernet Sauvignon grape bunches and its extractability during vinification. Am. J. Enol. Vitic., 53, 1-5.

Seaton J.C., Sugget A. \& Moir M., 1981. The role of sulphur compounds in beer flavour. In: Proceedings of the $18^{\text {th }}$ Congress of the European Brewery Convention, Copenhague, Denmark, 143-155.

Seaton J.C. \& Moir M., 1987. Sulfur compounds and their impact on beer flavor. In: Proceedings of the $21^{\text {th }}$ Congress of the European Brewery Convention, Madrid, Spain, 130-145.

Ségurel M. et al., 2005. Ability of possible precursors to release DMS during wine aging and in the conditions of heat alkaline treatment. J. Agric. Food Chem., 53, $2637-$ 2645.

Sharpe F.R. \& Laws D.R.J., 1981. The essential oil of hops. A review. J. Inst. Brew., 87, 96-107.

Shellhammer T.H., 2011. Hop aroma in beer: a comparison of brewers expectation versus sensory profiling. In: Proceedings of the $33^{\text {th }}$ Congress of the European Brewery Convention, 22-26 May 2011, Glasgow, Scotland, UK.

Starkenmann C., Niclass Y. \& Escher S., 2007. Volatile organic sulfur-containing constituents in Poncirus trifoliata (L.) Raf. (Rutaceae). J.Agric. Food Chem., 55, 4511-4517.

Starkenmann C. et al., 2015. Odorant volatile sulfur compounds in cat urine: occurrence of ( \pm )-3,7-dimethyloct-3-sulfanyl-6-en-1-ol and its cysteine conjugate precursor. Flavour Fragance. J., 30, 91-100.

Steinhaus M. \& Schieberle P., 2007. Transfer of the potent hop odorants linalool, geraniol and 4-methyl-4-sulfanyl2-pentanone from hops into beer. In: Proceedings of the $31^{\text {th }}$ Congress of the European Brewery Convention, 6-10 May 2007, Venice, Italy, 112-119.

Suggett A., Moir M. \& Seaton J.C., 1979. The role of sulphur compounds in hop flavour. In: Proceedings of the $17^{\text {th }}$ Congress of the European Brewery Convention, Berlin West, Germany, December 1979, 79-89.

Swiegers J.H., Bartowsky E.J., Henschke P.A. \& Pretorius I.S., 2005. Yeast and bacterial modulation of wine aroma and flavour. Aust. J. Grape Wine Res., 11, 139-173.

Swiegers J.H., Francis I.L., Herderich M.J. \& Pretorius I.S., 2006. Meeting consumer expectations through management in vineyard and winery. Aust. N.Z. Wine Ind. J., 21, 34-42.

Takabe K., Katagiri T.\& Tanaka J., 1970. Photodimerization of prenyl mercaptan. Tetrahedron Lett., 55, 4805-4806.

Takoi K. et al., 2009. Identification and characteristics of new volatile thiols derived from the hop (Humulus lupulus L.) cultivar Nelson Sauvin. J. Agric. Food Chem., 57, 2493-2502.

Tominaga T., Furrer A., Henry R. \& Dubourdieu D., 1998a. Identification of new volatile thiols in the aroma of Vitis vinifera L. var. Sauvignon Blanc wines. Flavour Fragance. J., 13, 159-162.

Tominaga T., Peyrot des Gachons C. \& Dubourdieu D., 1998b. A new type of flavor precursors in Vitis vinifera L. cv. Sauvignon Blanc: S-cysteine conjugates. J. Agric. Food Chem., 46, 5215-5219.

Tominaga T., Baltenweck-Guyot R., Peyrot des Gachons C. \& Dubourdieu D., 2000. Contribution of volatile thiols to the aromas of white wines made from several Vitis vinifera grape varieties. Am. J. Enol. Vitic., 51, 178-181.

Tominaga T., Niclass Y., Frérot E. \& Dubourdieu D., 2006. Stereoisomeric distribution of 3-mercaptohexan-1-ol and 3-mercaptohexyl acetate in dry and sweet white wines made from Vitis vinifera (Var. Sauvignon Blanc and Semillon). J. Agric. Food Chem., 54, 7251-7255.

Tran T.T.H., Kankolongo C.M.L. \& Collin S., 2015. Polyfunctional thiols in fresh and aged Belgian special beers: fate of hop S-cysteine conjugates. J. Am. Soc. Brew. Chem., 73, 61-70.

Vallarino J.G.etal., 2011.Biosynthesis of methoxypyrazines: elucidating the structural/functional relationship of two Vitis vinifera $O$-methyltransferases capable of catalyzing the putative final step of the biosynthesis of 3-alkyl-2methoxypyrazine. J. Agric. Food Chem., 59, 7310-7316.

Vandecan S.M.G. et al., 2010. Optimisation of specialty malt volatile analysis by headspace solid-phase microextraction in combination with gas chromatography and mass spectrometry. Anal. Chim. Acta, 671, 55-60.

Walker M.D., 1991. Formation and fate of sulphur voltailes in brewing. In: Proceedings of the $23^{\text {th }}$ Congress of the European Brewery Convention, Lisbon, Portugal, 521528. 